11

\title{
Динамика наведенного поглощения и нелинейно-оптический отклик в коллоидных квантовых точках $\mathrm{Ag}_{2} \mathrm{~S}$
}

\author{
() М.С. Смирнов ${ }^{1}$, О.В. Овчинников ${ }^{1}$, А.И. Звягин ${ }^{1}$, С.А. Тихомиров ${ }^{2}$, А.Н. Понявина ${ }^{2}$, В.А. Поведайло ${ }^{2}$, \\ Нгуен Тхань Бинь ${ }^{3}$, Фам Хонг Минь ${ }^{3}$ \\ ${ }^{1}$ Воронежский государственный университет, \\ 394018 Воронеж, Россия \\ ${ }^{2}$ Институт ффизики им. Б.И. Степанова НАН Беларуси, \\ 220072 Минск, Республика Беларусь \\ ${ }^{3}$ Институт фризики Вьетнамской академии наук и технологий, \\ Hanoi, Daotan, Thule, Badinh, 10, Vietnam \\ e-mail: Smirnov_M_S@mail.ru
}

Поступила в редакцию 26.11.2021 г.

В окончательной редакции 22.12.2021 г.

Принята к публикации 27.12.2021 г.

Рассмотрен механизм формирования нелинейно-оптического отклика коллоидных растворов квантовых точек $\mathrm{Ag}_{2} \mathrm{~S}$, пассивированных молекулами тиогликолевой кислоты. Методом $Z$-сканирования для $10 \mathrm{~ns}$ импульсов второй гармоники YAG:Nd-лазера показано возникновение нелинейного поглощения и нелинейной рефракции. Обнаружена насыщающаяся зависимость коэффициента поглощения при росте интенсивности падающего излучения. Сделано предположение об участии в процессе нелинейного поглощения локализованных состояний уровней структурных дефектов $\mathrm{Ag}_{2} \mathrm{~S}$, включая центр дефектной люминесценции, которое подтверждено методом фемтосекундной спектроскопии наведенного поглощения. Показано формирование широкой бесструктурной полосы наведенного поглощения в области 500-1000 nm, которая распадается на временной шкале сотен пикосекунд. Сделан вывод о том, что сигнал наведенного поглощения определяется быстрым (за доли пикосекунд) процессом захвата носителей заряда на локализованные состояния, которые в дальнейшем и определяют нелинейное поглощение $10 \mathrm{~ns}$ импульсов.

Ключевые слова: квантовые точки $\mathrm{Ag}_{2} \mathrm{~S}$, нелинейная рефракция, нелинейное поглощение, $Z$-сканирование, наведенное поглощение.

DOI: $10.21883 / \mathrm{OS} .2022 .04 .52282 .2964-21$

\section{Введение}

Исследование оптических свойств полупроводниковых коллоидных квантовых точек (КТ) является одним из актуальных направлений современной нанофотоники, что связано с широким спектром их практических приложений в медицине, биологии, фотокатализе и оптоэлектронике [1-4].

Ключевую роль в формировании люминесцентных свойств, а также нелинейно-оптического отклика накопительного характера играет динамика формирования и распада электронных возбуждений в КТ [5-9].

Общепринятые методы исследования спектров люминесценции дают информацию о типах излучательного распада электронных возбуждений и энергетике участвующих в нем структурно-примесных дефектов [9-11]. Использование техники $Z$-сканирования позволяет отслеживать процессы нелинейного поглощения и нелинейной рефракции, вызванные оптическими переходами между энергетическими состояниями размерного квантования, а также локализованными состояниями в КТ в поле лазерных импульсов различной длительности [12-14]. Однако детализация механизмов указанных процессов требует понимания кинетики формирования и распада электронных возбуждений, а также энергетической структуры участвующих состояний. Анализ кривых затухания люминесценции коллоидных КТ - весьма информативный прием $[9,10,15,16]$, дающий информацию о динамике излучательного распада возбуждений чаще всего в наносекундном временном диапазоне. Но в таком случае возникают сложности с исследованием безызлучательных процессов, в которых участвуют ловушки и центры безызлучательной рекомбинации. Существенное дополнение в картину динамики электронных возбуждений дает метод фемтосекундной спектроскопии наведенного поглощения („памп-проб“). Этот метод позволяет установить в реальном времени динамику фотопроцессов, обеспечивающих возбуждение КТ, его излучательный и безызлучательный распад $[7,17,18]$. В частности, появляется возможность выделить процессы, ответственные за формирования люминесценции и нелинейно-оптического отклика [8].

Размерная зависимость спектрально-люминесцентных свойств КТ определяет возможность настройки на резонанс между длиной волны падающего изучения и энергией соответствующего перехода, что позволяет в некоторых случаях снизить порог наступления нелинейнооптического отклика. Поэтому перспективным оказы- 
вается создание КТ, обладающих определенным набором локализованных состояний, заселение которых сопровождается обратным насыщающимся поглощением (ОНП) [12,19] или нелинейным преломлением из-за изменения заселенностей уровней энергии [20].

Для решения задач подобного рода интерес представляют коллоидные KT $\mathrm{Ag}_{2} \mathrm{~S}$. Сульфид серебра является соединением, обладающим заметной степенью нестехиометрии $[21,22]$. Эта особенность является одной из причин сложных люминесцентных свойств КТ $\mathrm{Ag}_{2} \mathrm{~S}$. В одних случаях наблюдают экситонную люминесценцию, в других - дефектную (trap state luminescence). Значительная нестехиометричность $\mathrm{Ag}_{2} \mathrm{~S}$ по атомам металла приводит к исходно высокой концентрации дефектов кристаллической структуры, часть которых может являться центрами излучательной рекомбинации [23,24], другая часть - локализованными состояниями, участвующими в поглощении [25]. Такие дефекты являются собственными. Нестехиометричность сульфида серебра сильно усложняет синтез и требует тщательного подбора условий кристаллизации, типов лигандов и т.д. Критерии однозначного отнесения типа свечения образцов различного размера и состава интерфейсов пока не установлены. Имеется значительное количество работ, в которых методом Z-сканирования обнаружено нелинейное поглощение для лазерных импульсов различной длительности [26,27]. Однако механизм нелинейного поглощения в коллоидных КТ $\mathrm{Ag}_{2} \mathrm{~S}$ остается предположительным - обсуждаются как двухфотонное поглощение, так и обратное насыщающееся поглощение [8,25].

Настоящая работа направлена на установление механизма формирования нелинейно-оптического отклика, закономерностей динамики электронных возбуждений, люминесценции и нелинейно-оптического отклика в КТ $\mathrm{Ag}_{2} \mathrm{~S}$, пассивированных молекулами тиогликолевой кислоты, с использованием метода фемтосекундной спектроскопии наведенного поглощения, времяразрешенной люминесцентной спектроскопии и метода $Z$-сканирования.

\section{Методы исследований и аппаратура}

Исследование абсорбционных свойств осуществляли с использованием спектрометра USB2000+ (OceanOptics, USA) с источником излучения USB-DT (OceanOptics, USA). Спектры люминесценции и кинетику затухания люминесценции КТ $\mathrm{Ag}_{2} \mathrm{~S}$ исследовали с помощью спектрофотометра USB2000+ и платы времякоррелированного однофотонного счета TimeHarp $\sim 260$ (PicoQuant Germany) с модулем ФЭУ PMC-100-20 (Becker\&Hickl Germany) с временным разрешением, составляющим $0.2 \mathrm{~ns}$. Кривые затухания люминесценции аппроксимировали теоретической кривой с использованием процедуры деконволюции с экспериментально измеренной функцией отклика аппаратуры.
Нелинейно-оптические свойства образцов коллоидных KT $\mathrm{Ag}_{2} \mathrm{~S}$ исследовали методикой Z-сканирования при использовании излучения второй гармоники $(532 \mathrm{~nm})$ $\mathrm{Nd}^{3+}$ :YAG-лазера (LS-2132UTF, LOTIS TII) с длительностью импульса $10 \mathrm{~ns}$ и частотой следования импульсов $1 \mathrm{~Hz}$ [28]. Методика основана на регистрации нормализованного пропускания образца в зависимости от расстояния до фокуса собирающей линзы $(f=300 \mathrm{~mm})$, через которую проходит гауссов лазерный пучок. Перемещением образца достигается сканирование по интенсивности падающего на него излучения. Диаметр перетяжки пучка в фокальной плоскости равнялся $50 \mu \mathrm{m}$. Данный комплекс управлялся компьютером. Образец помещали в кварцевую кювету толщиной $5 \mathrm{~mm}$ и перемещали вдоль оптической оси $Z$, проходя фокальную плоскость от линзы к калиброванному фотодиоду (ФД) при помощи моторизированного линейного транслятора 8MT50-200BS1-MEn1 (Standa), управляемого компьютеpom.

Схема Z-сканирования с открытой апертурой (ОА) позволяла измерять все прошедшее излучение для определения зависимости нормированного пропускания от положения образца на оси $Z$. Для наблюдения нелинейной рефракции использовали схему с закрытой апертурой (3А).

Динамику распада возбуждений исследовали методами фемтосекундной спектроскопии наведенного поглощения в видимой и ближней ИК областях и наносекундной кинетики люминесценции. В методе памппроб временное разрешение составляло $230 \mathrm{fs}$, что обеспечивалось лазерной системой, собранной по схеме задающий титан-сапфировый генератор-стрэтчерусилитель-компрессор. Для возбуждения образцов использовали вторую гармонику титан-сапфирового лазера. Пробным лучом выступало излучение фемтосекундного континуума, генерируемого первой гармоникой титан-сапфирового лазера в кювете с $\mathrm{H}_{2} \mathrm{O}$. Зондирование исследуемых объектов после возбуждения осуществляли в спектральном диапазоне 500-1000 nm. С коротковолновой стороны диапазон был ограничен областью сильного поглощения коллоидного раствора КТ $\mathrm{Ag}_{2} \mathrm{~S}$. C длинноволновой - областью чувствительности CCDматрицы. В спектральной области вблизи основной гармоники титан-сапфирового лазера (около $800 \mathrm{~nm}$ ) из-за высокой интенсивности в фемтосекундном континууме, определяемой непреобразованным излучением накачки, спектры наведенного поглощения не анализировались. Диапазон изменения задержек пробного луча составлял от 0.5 до $200 \mathrm{ps}$.

Размер КТ $\mathrm{Ag}_{2} \mathrm{~S}$ устанавливали с помощью просвечивающего электронного микроскопа (ПЭМ) Libra 120 (CarlZeiss, Germany) с ускоряющим напряжением $120 \mathrm{kV}$. 


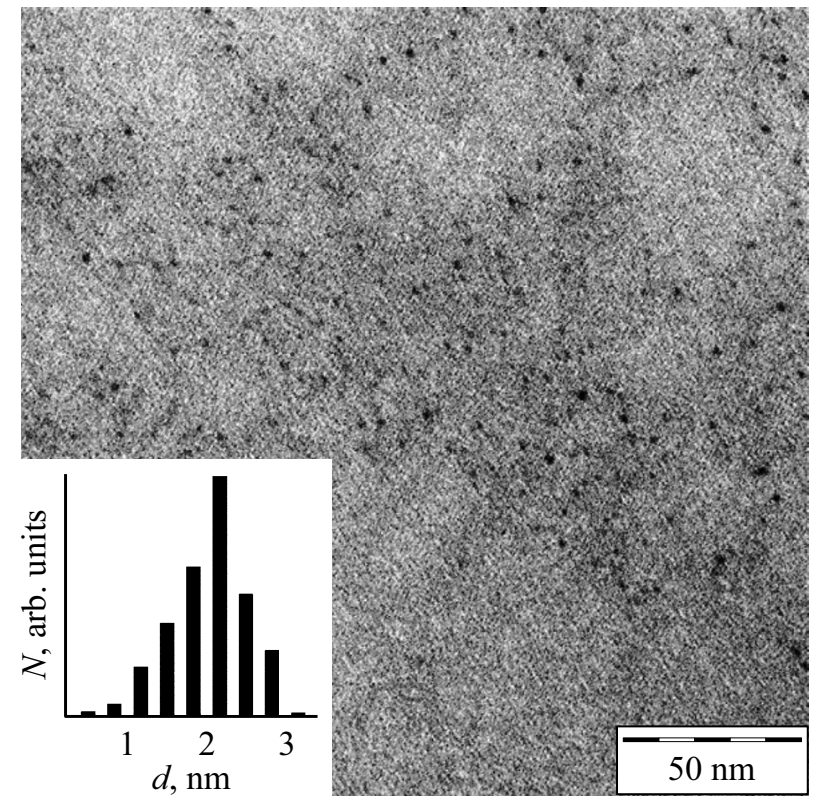

Рис. 1. ТЕМ-изображение и гистограмма распределения по размерам KT $\mathrm{Ag}_{2} \mathrm{~S}$.

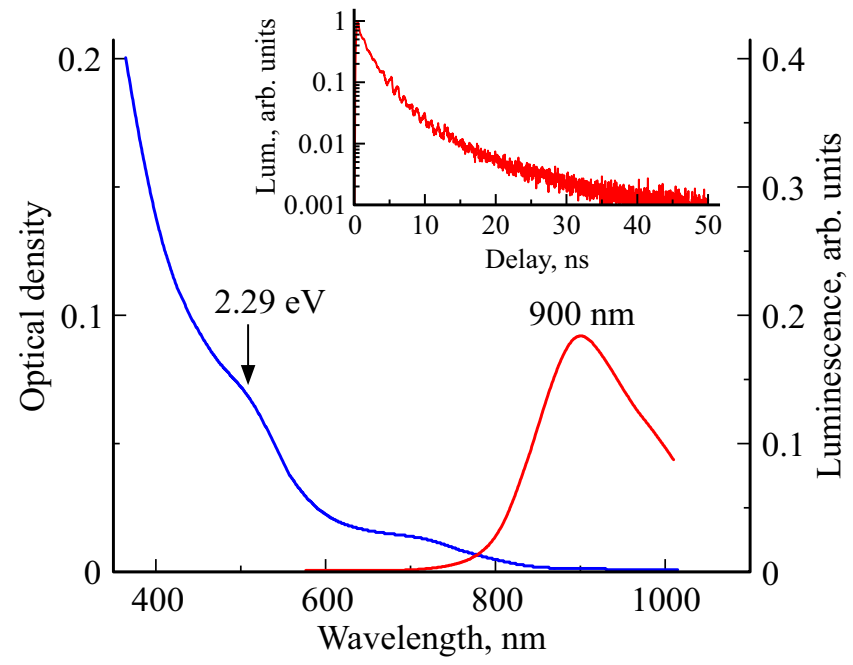

Рис. 2. Спектры поглощения и люминесценции КТ $\mathrm{Ag}_{2} \mathrm{~S}$. На врезке кинетика затухания люминесценции на длине волны $900 \mathrm{~nm}$.

\section{Исследованные образцы}

Методика синтеза КТ $\mathrm{Ag}_{2} \mathrm{~S}$, пассивированных тиогликолевой кислотой (TGA), подробно описана в [29]. Прекурсор $\mathrm{Ag}^{+} / \mathrm{TGA}$ получали смешиванием $\mathrm{AgNO}_{3}$ $(0.6 \mathrm{mmol})$ и TGA $(0.6 \mathrm{mmol})$. Нитрат серебра растворяли в воде $(100 \mathrm{ml})$, добавляли тиогликолевую кислоту и $\mathrm{NaOH}(1 \mathrm{M})$, доводя уровень $\mathrm{pH}$ до 10 . В приготовленный раствор $\mathrm{Ag}^{+} / \mathrm{TGA}$ постепенно добавляли $50 \mathrm{ml}$ водного раствора $\mathrm{Na}_{2} \mathrm{~S}(0.3 \mathrm{mmol})$. Полученные таким способом КТ осаждали этанолом, центрифугировали и повторно растворяли в дистиллированной воде.
Анализ ПЭМ-изображений позволил установить дисперсию размеров КТ в ансамбле и определить их средний размер (рис. 1). Показано формирование ансамблей нанокристаллов со средним размером $2.2 \mathrm{~nm}$ с распределением по размеру в диапазоне $20-30 \%$.

\section{Результаты и их обсуждение}

На рис. 2 представлены спектры оптического поглощения и люминесценции образца КT $\mathrm{Ag}_{2} \mathrm{~S}$. Широкий бесструктурный спектр линейного поглощения расположен в области от 200 до $950 \mathrm{~nm}$ и обусловлен вкладом как от экситонного поглощения нанокристаллов, отличающихся размерами, так и от поглощения локализованными состояниями нестехиометрического соединения $\mathrm{Ag}_{2} \mathrm{~S}[8,11]$. Характерный перегиб, расположенный около $2.29 \mathrm{eV}$, связан с экситонным поглощением и смещен в коротковолновую сторону относительно края поглощения массивного $\mathrm{Ag}_{2} \mathrm{~S}(1.0 \mathrm{eV})$, что является проявлением квантового ограничения. Оценка среднего размера КТ $\mathrm{Ag}_{2} \mathrm{~S}$, выполненная на основе установленного значения энергии экситонного поглощения с использованием формулы Y. Kayanuma [30]

$$
E_{\mathrm{exc}}=E_{g}^{\mathrm{bulk}}+\frac{\hbar^{2} \pi^{2}}{2 \mu R^{2}}+\frac{1.8 e^{2}}{\varepsilon R}-0.248 E_{\mathrm{Ry}}^{*},
$$

где $E_{\mathrm{exc}}-$ энергия пика экситонного поглощения KT, $E_{g}^{\text {bulk }}$ - ширина запрещенной зоны массивного кристалла $\operatorname{Ag}_{2} \mathrm{~S}, \mu=\frac{m_{e}^{*}-m_{h}^{*}}{m_{e-}^{*}+m_{h+}^{*}}-$ приведенная эффективная масса электрона и дырки, $e-$ заряд электрона, $\varepsilon-$ диэлектрическая проницаемость $\mathrm{Ag}_{2} \mathrm{~S}$, $E_{\mathrm{Ry}}^{*}=e^{4} / 2 \varepsilon^{2} \hbar^{2}\left(\frac{1}{m_{e-}^{*}}+\frac{1}{m_{h+}^{*}}\right)-$ эффективная энергия Ридберга, дала величину среднего размера КТ $\mathrm{Ag}_{2} \mathrm{~S}$ порядка $2.0 \mathrm{~nm}$.

Максимум полосы люминесценции коллоидных КТ $\mathrm{Ag}_{2} \mathrm{~S}$ расположен в ИК области при $900 \mathrm{~nm}$. Пик люминесценции сдвинут относительно пика экситонного поглощения в длинноволновую область на $0.91 \mathrm{eV}$, что указывает на дефектный характер наблюдаемой люминесценции [11]. Таким образом, исследуемые КТ $\mathrm{Ag}_{2} \mathrm{~S}$ содержат как минимум один тип локализованных состояний - центр люминесценции. Однако заметный длинноволновый „хвост“ в спектре поглощения, а также значительная полуширина полосы люминесценции КТ $\mathrm{Ag}_{2} \mathrm{~S}$ указывают на заметную плотность локализованных состояний и их сложную структуру.

На основании исследования размерной зависимости стоксова сдвига и теоретических оценок величины размерного эффекта для уровней размерного квантования дырок и электронов показано, что люминесценция возникает в результате рекомбинации локализованного на центре свечения электрона с дыркой [11].

Для коллоидных $\mathrm{KT} \mathrm{Ag}_{2} \mathrm{~S}$ в полосе дефектной люминесценции обнаружен сложный закон затухания (рис. 2, врезка). Причины мультиэкспоненциального закона зату-

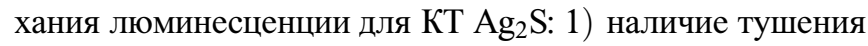



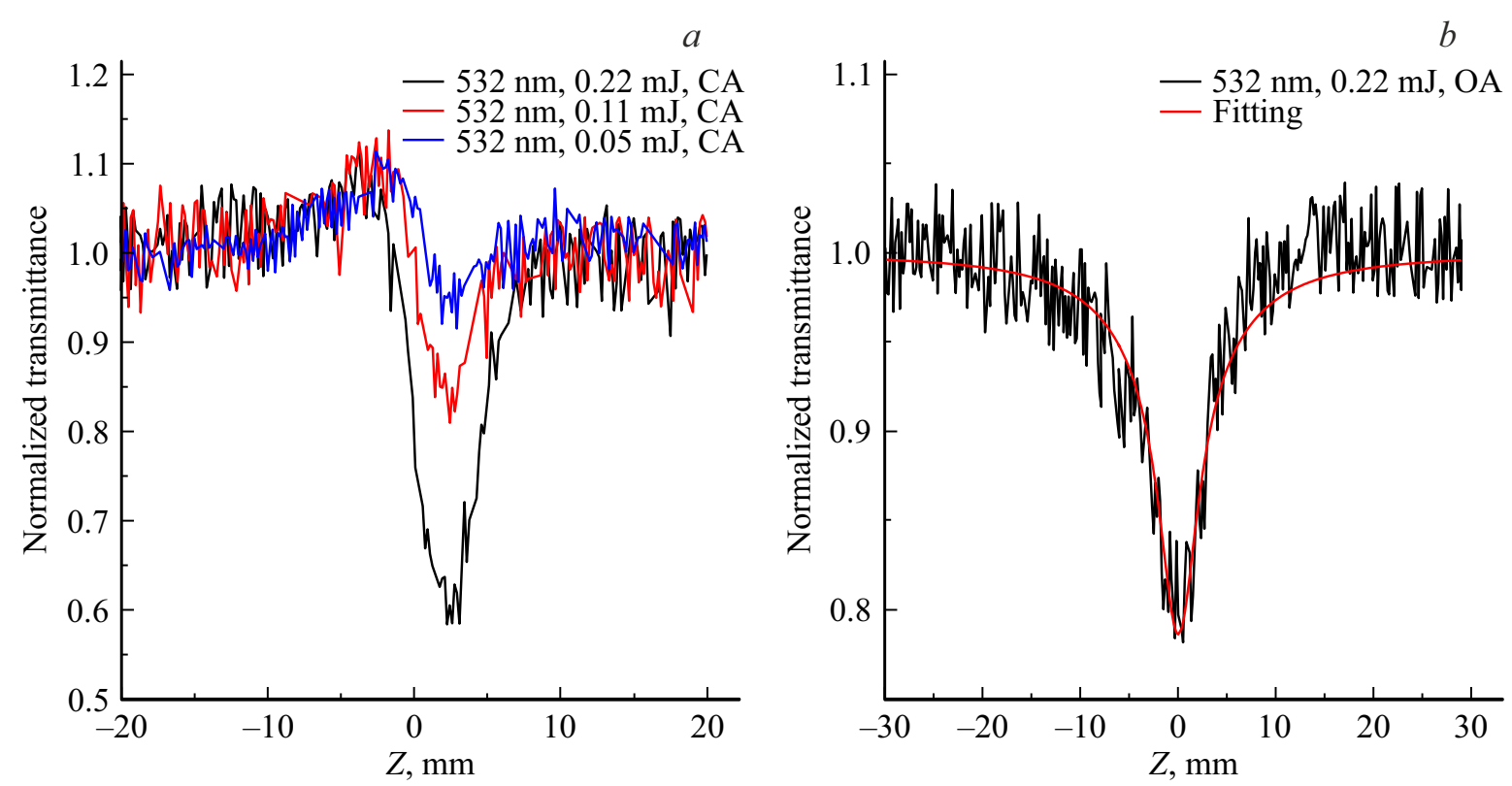

Рис. 3. Z-сканы КT $\operatorname{Ag}_{2} \mathrm{~S}$ в геометрии с закрытой $(a)$ и открытой $(b)$ апертурой на длине волны 532 nm, длительность импульсов $10 \mathrm{~ns}$.

молекулами растворителя или собственными дефектами [31] и распределение по константам скоростей тушения; 2) дисперсия КТ по размерам и уровней центров свечения по энергиям. В работе [11] показано, что вариация размера $\mathrm{KT} \mathrm{Ag}_{2} \mathrm{~S}$ не приводит к заметному изменению среднего времени жизни люминесценции. Таким образом, дисперсия по размеру КТ не должна приводить к распределению по константам скоростей для различных КТ в образце и сложному закону затухания люминесценции, а основной причиной неэкспоненциального закона затухания является распределение по числу тушителей люминесценции для КТ в образце $[32,33]$. Кинетика затухания люминесценции с учетом распределения по числу тушителей в модели Тасhіа имеет вид [31]:

$$
\begin{aligned}
I_{\text {lum }} & \sim N^{*}(t)=\sum_{n=0}^{\infty} N_{n}^{*}(t) \\
& \left.=N^{*}(0) \exp \left[-\langle k\rangle t-\langle m\rangle\left(1-\exp \left[-k_{m} t\right]\right)\right)\right],
\end{aligned}
$$

где $\langle k\rangle-$ средняя скорость затухания люминесценции КТ в отсутствие тушителей, $\langle m\rangle-$ среднее число тушителей люминесценции, приходящееся на одну КТ в образце, $k_{m}-$ константа тушения одним центром тушения в КТ.

Кроме того, распределение константы рекомбинации по величине может быть обусловлено также и локальной природой центра дефектной люминесценции (в отличие от экситонной люминесценции, где возбуждение делокализовано по всему объему КТ), расположенного в каждой КТ ансамбля в различном окружении. Таким образом, неэкспоненциальная кинетика затухания люми-

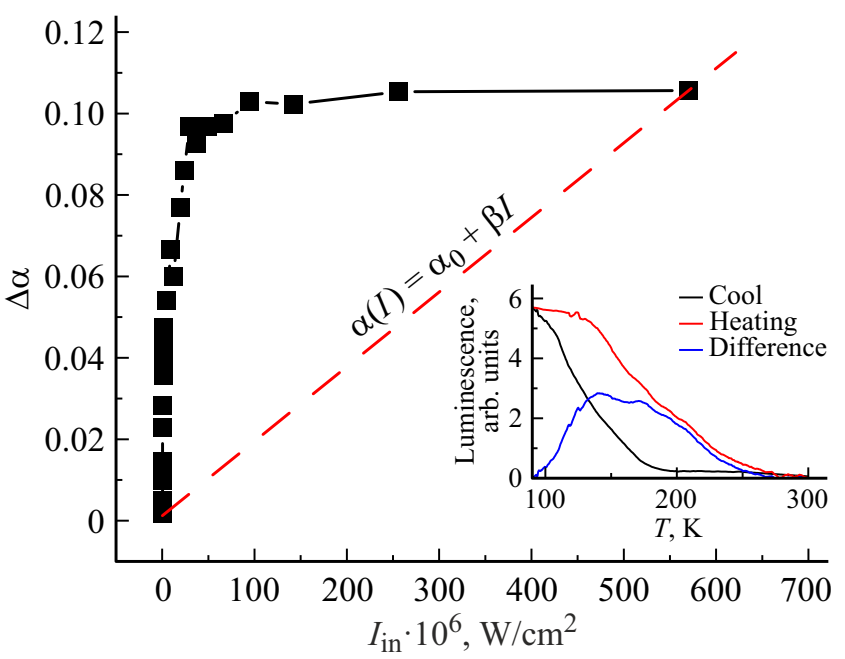

Рис. 4. Зависимость показателя поглощения растворов КТ $\mathrm{Ag}_{2} \mathrm{~S}$ от интенсивности зондирующего излучения. На врезке температурные зависимости интегральной интенсивности фотолюминесценции при исследовании термостимулированной люминесценции.

несценции является неотьемлемым свойством дефектной люминесценции.

Рассмотрим нелинейно-оптический отклик КТ $\mathrm{Ag}_{2} \mathrm{~S}$ (рис. 3), исследованный методикой Z-сканирования.

Кривые $Z$-сканирования образца $\mathrm{KT} \mathrm{Ag}_{2} \mathrm{~S}$ в закрытой апертуре имеют асимметричный профиль (рис. $3, a$ ) для энергий лазерного импульса от 0.05 до $0.22 \mathrm{~mJ}$ и соответствуют дефокусировке. Кроме того, для $Z=0$ нормализованное пропускание образца меньше 1 , что указывает на одновременное проявление эффекта ОНП. 
В работе [34] на основании решения уравнения тепловой диффузии показано, что в условиях проводимого эксперимента тепловая дефокусировка не наблюдается или имеет величину меньше порога регистрации аппаратуры, а нелинейная рефракция обусловлена заполнением локализованных состояний структурных дефектов под воздействием лазерного излучения.

Кривые Z-сканирования, полученные в геометрии с открытой апертурой, демонстрировали симметричный провал (рис. $3, b)$ в нормализованном пропускании около фокальной плоскости $(Z=0)$. Низкий порог проявления наблюдаемой нелинейности указывает на ОНП, возникающее в результате двухквантовых переходов в КТ $\mathrm{Ag}_{2} \mathrm{~S}$ через реальные уровни структурных дефектов. Таким условиям могут удовлетворять центры дефектной люминесценции.

На участие локализованных состояний в формировании ОНП также указывает вид зависимости коэффициента поглощения от интенсивности падающего на образец излучения, $\Delta \alpha(I)$ (рис. 4). Эту зависимость получали из кривой $Z$-сканирования (зависимость нормализованного пропускания от положения образца $Z$ ), перестроенной с учетом зависимости интенсивности в сфокусированном гауссовом пучке лазерного излучения от $Z$. Видно, что коэффициент поглощения при росте интенсивности повышается до некоторого уровня и далее практически не изменяется. Такое поведение коэффициента поглощения характерно для ОНП с участием реальных уровней. Выход на плато определяется тем, что насыщается первая ступень в двухквантовом переходе [35], ответственном за нелинейное поглощение. При двухфотонном поглощении коэффициент является линейной функцией интенсивности падающего излучения $\left(\alpha=\alpha_{0}+\beta I\right.$, рис. 4, штрихи).

Коэффициент нелинейного поглощения $\beta$ определяли из аппроксимации экспериментальных $Z$-сканов теоретической кривой [36]

$$
T(z)=1+\frac{4 x}{\left(x^{2}+9\right)\left(x^{2}+1\right)} \Delta \Phi-\frac{2\left(x^{2}+3\right)}{\left(x^{2}+9\right)\left(x^{2}+1\right)} \Delta \Psi .
$$

Здесь $x=z / z_{0}, z_{0}=0.5 k\left(w_{0}\right)^{2}, k=2 \pi / \lambda, w_{0}$ - радиус пучка на уровн $1 / e^{2}$ пространственного распределения интенсивности в фокальной плоскости, $\lambda$ - длина волны излучения, $\Delta \Phi=k \gamma I_{0} L_{\mathrm{ef}}$ и $\Delta \Psi=\beta I_{0} L_{\mathrm{ef}} / 2$ - параметры, описывающие фазовый сдвиг вблизи фокальной точки (эти параметрысоответствуют нелинейному преломлению и нелинейному поглощению соответственно), $\gamma$ нелинейный показатель преломления, $\beta$ - коэффициент нелинейного поглощения, $I_{0}-$ интенсивность лазерного излучения в перетяжке, $L_{\mathrm{ef}}=[1-\exp (-\alpha L)] / \alpha-$ эффективная толщина образца, $\alpha-$ коэффициент линейного поглощения, $L$ - толщина образца. Она оказалась равной $3.0 \cdot 10^{-9} \mathrm{~cm} / \mathrm{W}$. Однако эта величина оказывается сильно заниженной, поскольку зависимость нормализованного пропускания от координаты фактически

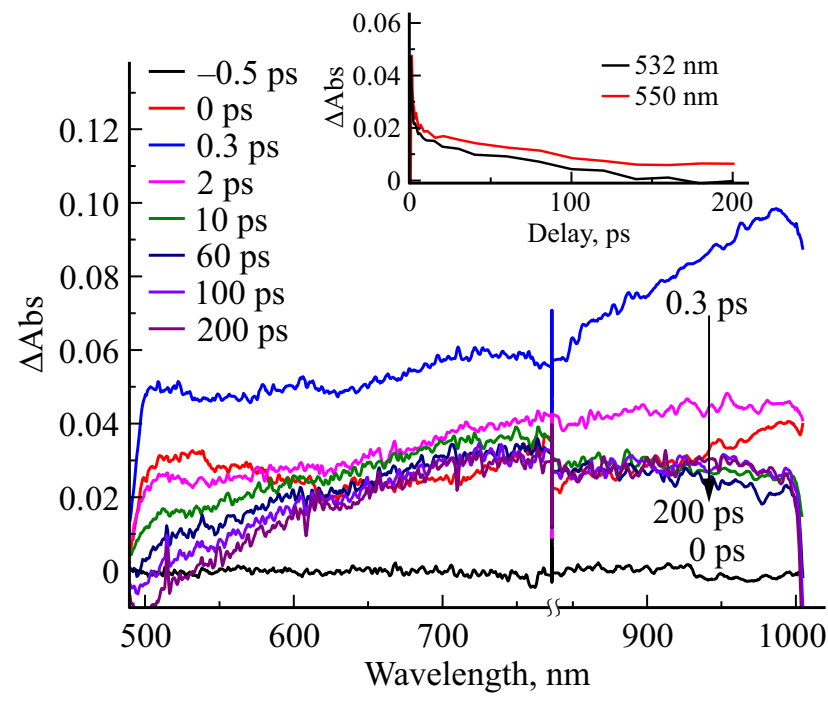

Рис. 5. Спектры наведенного поглощения коллоидных КТ $\mathrm{Ag}_{2} \mathrm{~S}$ для энергии накачивающего импульса $2 \mathrm{mkJ}$ и кинетика релаксации наведенного поглощения для некоторых длин волн.

аппроксимируется кривой для двухфотонного поглощения, или, что тоже самое, зависимость $\Delta \alpha(I)$ аппроксимируют штриховой прямой. Такой подход будет давать всегда заниженное значение $\beta$. Более того, это значение будет тем меньше, чем больше интенсивность пробного пучка в перетяжке. Значение нелинейного коэффициента поглощения, полученное для невысокой интенсивности (начальный участок кривой $\Delta \alpha(I))$ дает величину примерно на 2 порядка больше, т.е. $\beta \sim 3.0 \cdot 10^{-7} \mathrm{~cm} / \mathrm{W}$. Авторы работы [25] исследовали нелинейное поглощение КТ $\mathrm{Ag}_{2} \mathrm{~S}$ с экситонной люминесценцией и экситонным переходом в поглощении при $1.7 \mathrm{eV}$ и определили величину коэффициента нелинейного поглощения $\beta=5.5 \cdot 10^{-8} \mathrm{~cm} / \mathrm{W}$ (интенсивность в перетяжке $\left.0.3 \cdot 10^{9} \mathrm{~W} / \mathrm{cm}^{2}\right)$, что несколько меньше установленного нами значения $\left(\beta \sim 3.0 \cdot 10^{-7} \mathrm{~cm} / \mathrm{W}\right)$. Однако, несмотря на большой коэффициент нелинейного поглощения, сделан вывод о двухфотонных переходах через виртуальное состояние [25].

Разделение механизма нелинейного поглощения на основании только сравнения коэффициентов $\beta$ имеет предположительный характер. Главным доводом в пользу участия локализованных состояний в формировании нелинейного поглощения являются закономерности фемтосекундной динамики электронных возбуждений для коллоидных КТ $\mathrm{Ag}_{2} \mathrm{~S}$. Спектры наведенного поглощения коллоидных КT $\mathrm{Ag}_{2} \mathrm{~S}$ в спектральном диапазоне 500-1000 nm и временной шкале до $200 \mathrm{ps}$ представлены на рис. 5. Отметим наиболее важные закономерности спектров наведенного поглощения КТ $\mathrm{Ag}_{2} \mathrm{~S}$. Во-первых, все слабовыраженные полосы в спектральной области 500-1000 nm формируются практически без задержки за субпикосекундные времена в соответствии с аппаратной функцией спектрометра. Во- 
вторых, формируется широкая бесструктурная полоса наведенного поглощения во всей спектральной области регистрации, которая постепенно затухает, слабо меняя свою форму. В-третьих, полоса просветления в области экситонного поглощения, быстрое появление которой при возбуждении свойственно КТ [17], непосредственно после возбуждения образцов в рассматриваемом случае практически не проявляется.

Напомним, что для массивных полупроводниковых кристаллов бесструктурный спектр наведенного поглощения, интенсивность которого увеличивается с ростом длины волны, обусловлен поглощением свободными носителями заряда и возбуждением их внутри зоны проводимости. В случае КТ такое объяснение наблюдающегося сигнала встречает затруднения, поскольку вместо непрерывной зоны проводимости наблюдается дискретный энергетический спектр электронов. В таком случае в наведенном поглощении следовало бы ожидать присутствия селективных полос, обусловленных переходами между этими дискретными уровнями. Однако такие полосы в спектрах наведенного поглощения отсутствуют. Наиболее реалистичной причиной наблюдающейся бесструктурной полосы в спектре наведенного поглощения (аналогично, как и для стационарного поглощения) является примесное поглощение собственными дефектами КТ $\mathrm{Ag}_{2} \mathrm{~S}$.

Итак, для KT $\mathrm{Ag}_{2} \mathrm{~S}$ обнаружено наведенное поглощение в широкой спектральной области от 500 до $1000 \mathrm{~nm}$. При этом кинетика релаксации этого поглощения описывается, по крайней мере, двухэкспоненциальными зависимостями с временными постоянными порядка 2 и $>1000$ ps и различающимися амплитудами в зависимости от спектральной области зондирования. Поскольку обе компоненты релаксации сигнала наведенного поглощения многократно превышают длительность возбуждающих импульсов, очевидно, что оно обусловлено переходами с участием реальных состояний. Этот факт крайне важен для понимания механизмов накопительных нелинейностей, исследуемых методикой $Z$-сканирования в $\mathrm{KT} \mathrm{Ag}_{2} \mathrm{~S}$. Кроме того, необходимо напомнить, что в нестационарных спектрах на малых временных задержках отсутствует полоса наведенного просветления, которую можно было бы сопоставить с экситонной полосой поглощения в стационарном спектре поглощения. Эти факты указывают на то, что имеет место быстрый захват возбужденных носителей заряда на локализованные состояния (минуя экситонное), что определяется заметной концентрацией дефектов, существование которых также отмечено при анализе спектров оптического поглощения и фотолюминесценции.

Более того, по всему спектру преобладает наведенное поглощение, которое определяется переходами с участием структурных дефектов, в том числе и центров люминесценции, для которых показана возможность прямого возбуждения с участием глубоких состояний уровней размерного состояния для дырок. Излучательная рекомбинация на центрах люминесценции имеет характерное время 2-300 ns. Конкуренцию с процессом излучения составляет захват носителей заряда на локализованные состояния, обнаруженные методикой термостимулированной люминесценции, с энергиями ионизации $0.05-0.08$ и $0.1-0.12 \mathrm{eV}$ (рис. 4, врезка) [37]. Эти уровни, по-видимому, также участвуют в формировании как полосы наведенного поглощения, так и сигнала нелинейной рефракции в методике $Z$-сканирования.

Таким образом, схема состояний и фотопроцессов с их участием сложна и включает в себя не только уровни размерного квантования и центр дефектной люминесценции, но и локализованные состояния, которые напрямую не участвуют в люминесценции, но определяют быстрый захват экситона, формирование нелинейного поглощения и нелинейной рефракции, а также неэкспоненциальный закон затухания люминесценции.

\section{Заключение}

В настоящей работе представлены результаты исследований динамики распада возбуждений, определяющей нелинейно-оптический отклик коллоидных КТ $\mathrm{Ag}_{2} \mathrm{~S}$, покрытых молекулами тиогликолевой кислоты и обладающих дефектной люминесценцией. Методом Zсканирования для $10 \mathrm{~ns}$ импульсов второй гармоники YAG:Nd-лазера $(532 \mathrm{~nm})$ в геометрии с закрытой и открытой апертурами обнаружены нелинейное поглощение и нелинейная рефракция. Из данных о зависимости нормализованного пропускания образца от расстояния до фокуса собирающей линзы получена зависимость коэффициента поглощения от падающей на образец интенсивности. На основе обнаруженного насыщения этой зависимости сделано предположение, что нелинейное поглощение определяется двухквантовыми переходами через реальные состояния, поскольку для двухфотонных переходов через виртуальные состояния эта зависимость представляет собой наклонную прямую. Предположено, что нелинейное поглощение определяется двухквантовыми переходами через локализованные состояния уровней структурных дефектов $\mathrm{Ag}_{2} \mathrm{~S}$, включая центр дефектной люминесценции. Доказательство механизма нелинейного поглощения осуществлено методом фемтосекундной спектроскопии наведенного поглощения. Обнаружено формирование широкой бесструктурной полосы наведенного поглощения и отсутствие просветления на малых временных задержках в области экситонного поглощения. Сделан вывод о быстром захвате носителей на локализованные состояния за субпикосекундные времена. Обнаружено, что кинетика релаксации наведенного поглощения содержит как минимум две компоненты с постоянными времени, значительно превышающими длительность используемых импульсов. Сделан вывод, что первоначальный сигнал наведенного поглощения определяется быстрым (за доли пикосекунд) процессом захвата носителей заряда на локализованные состояния, 
которые в дальнейшем и определяют нелинейное поглощение $10 \mathrm{~ns}$ импульсов.

\section{Благодарности}

Работа поддержана грантами РФФИ (№ 20-5281005 ЕАПИ_т), БРФФИ (№ Ф20ЕA-006), VANT (No. QTRU05.02/21-23).

\section{Конфликт интересов}

Авторы заявляют, что у них нет конфликта интересов.

\section{Список литературы}

[1] K.A.A. Mary, N.V. Unnikrishnan, Reji Philip. APL Mater., 2, 076104 (2014). DOI: 10.1063/1.4886276

[2] A.I. Zvyagin, M.S. Smirnov, O.V. Ovchinnikov, A.S. Selyukov. Bull. Lebedev Phys. Inst., 46, 93 (2019). DOI: $10.3103 / \mathrm{S} 1068335619030059$

[3] A.S. Selyukov, A.G. Vitukhnovskii, V.S. Lebedev, A.A. Vashchenko, R.B. Vasiliev, M.S. Sokolikova. J. Exp. Theor. Phys., 120, 595 (2015). DOI: $10.1134 / \mathrm{S} 1063776115040238$

[4] B. Zhu, F. Wang, Ch. Liao, H. Zhang, J. Zhang, Y. Cui, Y. Ye, Y. Gu. Opt. Express, 27, 1777 (2019). DOI: $10.1364 /$ OE.27.001777

[5] O.V. Ovchinnikov, M.S. Smirnov, B.I. Shapiro, T.S. Shatskikh, A.S. Perepelitsa, N.V. Korolev. Semiconductors, 49, 373 (2015). DOI: 10.1134/S1063782615030173

[6] Q. Chang, Y. Gao, X. Liu, etal. IOP Conf. Ser: Earth Environ. Sci., 186, 012076 (2018). DOI: 10.1088/17551315/186/4/012076

[7] P. Kambhampati. J. Phys. Chem. C, 115, 22089 (2011). DOI: $10.1021 / \mathrm{jp} 2058673$

[8] T.S. Kondratenko, A.I. Zvyagin, M.S. Smirnov, I.G. Grevtseva, A.S. Perepelitsa, O.V. Ovchinnikov. J. Lumin., 208, 193 (2019). DOI: 10.1016/j.jlumin.2018.12.042

[9] E.N. Bodunov, A.L. Simoes Gamboa. Semiconductors, 53, 2133 (2019). DOI: 10.1134/S1063782619120078

[10] O.V. Ovchinnikov, M.S. Smirnov, N.V. Korolev, P.A. Golovinski, A.G. Vitukhnovsky. J. Lumin., 179, 413 (2016). DOI: $10.1016 /$ j.jlumin.2016.07.016

[11] M.S. Smirnov, O.V. Ovchinnikov. J. Lumin., 227, 117526 (2020). DOI: 10.1016/j.jlumin.2020.117526

[12] H. Aleali, N. Mansour. Optik, 127, 2485 (2016). DOI: 10.1016/j.ijleo.2015.11.146

[13] K. Kannan, L.G. Prasad, S. Agilan, N. Muthukumarasamy. Optik, 170, 10 (2018). DOI: 10.1016/j.ijleo.2018.05.078

[14] T.S. Kondratenko, M.S. Smirnov, O.V. Ovchinnikov, A.I. Zvyagin, R.A. Ganeev, I.G. Grevtseva. Optik, 200, 163391 (2020). DOI: $10.1016 /$ j.ijleo.2019.163391

[15] A.V. Katsaba, S.A. Ambrozevich, A.G. Vitukhnovsky, V.V. Fedyanin, A.N. Lobanov, V.S. Krivobok, R.B. Vasiliev, I.G. Samatov. J. Appl. Phys., 113, 184306 (2013). DOI: $10.1063 / 1.4804255$

[16] D. Ruiz, B. del Rosal, M. Acebrón, C. Palencia, C. Sun, J. Cabanillas-González, M. López-Haro, A.B. Hungría, D. Jaque, B.H. Juarez. Adv. Funct. Mater., 27, 1604629 (2017). DOI: 10.1002/adfm.201604629
[17] M.S. Smirnov, O.V. Buganov, S.A. Tikhomirov, O.V. Ovchinnikov. Physica E, 118, 113898 (2020). DOI: 10.1016/j.physe.2019.113898

[18] M.S. Smirnov, O.V. Buganov, S.A. Tikhomirov, O.V. Ovchinnikov. J. Lumin., 232, 117794 (2021). DOI: $10.1016 /$ j.jlumin.2020.117794

[19] J.V. Antony, J.J. Pillai, Ph. Kurian, V.P.N. Nampoori, G.E. Kochimoolayil. New J. Chem., 41, 3524 (2017). DOI: 10.1039/C6NJ03665A

[20] N. Venkatram, R.S.S. Kumar, D.N. Rao. J. Appl. Phys., 100, 074309 (2006). DOI: 10.1063/1.2354417.

[21] S.I. Sadovnikov, A.I. Gusev, A.A. Rempel. Phys. Chem. Chem. Phys., 17, 12466 (2015). DOI: 10.1039/C5CP00650C

[22] S.I. Sadovnikov, A.I. Gusev. J. Mater. Chem. A, 5, 17676 (2017). DOI: 10.1039/C7TA04949H

[23] A. Pourahmad. Superlattices Microstruct., 52, 276 (2012). DOI: 10.1016/j.spmi.2012.05.009

[24] R. Tang, J. Xue, B. Xu, D. Shen, G.P. Sudlow, S. Achilefu. ACS Nano, 9, 220 (2015). DOI: 10.1021/nn5071183

[25] W.J. Mir, A. Swarnkar, R. Sharma, A. Katti, K.V. Adarsh, A. Nag. J. Phys. Chem. Lett., 6, 3915 (2015). DOI: 10.1021/acs.jpclett.5b01692

[26] K.A.A. Mary, N.V. Unnikrishnan, R. Philip. Mater. Res. Bull., 70, 321 (2015). DOI: 10.1016/j.materresbull.2015.04.034

[27] M. Dehghanipour, M. Khanzadeh, M. Karimipour, M. Molaei. Opt. Laser Technol., 100, 286 (2018). DOI: $10.1016 /$ j.optlastec.2017.10.023

[28] R.A. Ganeev, A.I. Zvyagin, O.V. Ovchinnikov, M.S. Smirnov. Dyes. Pigm., 149, 236 (2018). DOI: $10.1016 /$ j.dyepig.2017.09.063

[29] O.V. Ovchinnikov, I.G. Grevtseva, M.S. Smirnov, T.S. Kondratenko. J. Lumin., 207, 626 (2019). DOI: $10.1016 /$ j.jlumin.2018.12.019

[30] Y. Kayanuma. Phys. Rev. B, 38, 9797 (1988). DOI: 10.1103/PhysRevB.38.9797

[31] M. Tachiya. J. Chem. Phys., 76, 340 (1982). DOI: $10.1063 / 1.442728$

[32] R. Koole, B. Luigjes, M. Tachiya, R. Pool, T.J.H. Vlugt, C. de Mello Donegá, A. Meijerink, D. Vanmaekelbergh. J. Phys. Chem. C, 111, 11208 (2007). DOI: $10.1021 / \mathrm{jp} 072407 \mathrm{x}$

[33] E.N. Bodunov, A.L. Simões Gamboa. Semiconductors, 52, 587 (2018). DOI: 10.1134/S1063782618050044

[34] A.I. Zvyagin, T.A. Chevychelova, I.G. Grevtseva, M.S. Smirnov, A.S. Selyukov, O.V. Ovchinnikov, R.A. Ganeev. J. Russ. Laser Res., 41, 670 (2020). DOI: $10.1007 / \mathrm{s} 10946-020-09923-4$

[35] L.W. Tutt, T.F. Bogess. Prog. Quant Electr., 17, 299 (1993). DOI: 10.1016/0079-6727(93)90004-S

[36] X. Liu, S. Guo, H. Wang, L. Hou. Opt. Commun., 197, 431 (2001). DOI: 10.1016/S0030-4018(01)01406-7

[37] A.S. Perepelitsa, M.S. Smirnov, O.V. Ovchinnikov, A.N. Latyshev, A.S. Kotko. J. Lumin., 198, 357 (2018). DOI: 10.1016/j.jlumin.2018.02.009 